\title{
TRANSESOPHAGEAL ECHOCARDIOGRAPHY AND AORTIC VALVE SURGERY
}

André Y. Denault MD FRCPC, Baqir Qizilbash MD, Pierre Couture MD FRCPC, Raymond Cartier MD FRCSC

Departments of Anesthesiology and Cardiac Surgery, Montreal Heart Institute, 5000 Bélanger Street, Montreal, Quebec H1T1C8

INTRODUCTION: Intraoperative transesophageal echocardiography (TEE) is currently being used frequently during aortic valve surgery across North America and is classified as a class II indication as stated by the ASE/SCA. We describe 10 years experience on the impact of TEE performed by anesthesiologists in the operating room for patients undergoing aortic valve surgery.

METHODS: Following approval from the Ethic and Research Committee on the use of a TEE database for quality control, 879 consecutive TEE examinations performed from November 1993 to October 2003 in which the aortic valve was repaired, replaced alone or in combination with other procedures (complex procedures) at two institutions were studied. All examinations were performed or supervised by anesthesiologists who had advanced training in TEE and certification from the National Board of Echocardiography. The exams were entered in a database and a second anesthesiologist then reviewed all the data sheets. The impact of TEE was classified in three categories. A surgical impact included: 1. return to cardiopulmonary bypass for hemodynamic reasons; 2. revision of the procedure due to inadequate surgical results; 3. modification of the site of aortic cannulation; 4. modification of the planned surgical procedure including cancellation or addition of surgical acts. A medical impact included the addition or alteration of inotropic or vasodilator medications or volume therapy or confirmation of a previous diagnosis. Finally we also noted the role of TEE in guidance for the placement of various surgical cannulae including intra-aortic balloon pumps, retrograde cardioplegia cannulae, venous bypass cannulae, or catheters and modification of miscellaneous other therapies.

RESULTS: A total of 177 (20.1\%) modifications were reported in the 879 examinations: $9.6 \%$ of modifications were surgical, $9.2 \%$ were medical and there were $1.4 \%$ other modifications. In aortic valve replacement alone, surgical changes occurred in $8.2 \%$ and medical changes occurred in $5.5 \%$ of cases. In complex procedures modification of therapy occurred more frequently (33.1\%).

CONCLUSION: The impact of TEE in aortic valve surgery is significant and the impact is closer to category I than II indication.

Supported by the "Fondation de l'Institut de Cardiologie de Montréal", the "Fonds de la recherche en santé du Québec". 